\title{
Three-dimensional surgical accuracy between virtually planned and actual surgical movements of the maxilla in two-jaw orthognathic surgery
}

Mihee Hong ${ }^{\mathrm{a}}$ (1)

Myung-Jin Kim ${ }^{\mathrm{b}}$

Hye Jung Shin ${ }^{b}$

Heon Jae Cho ${ }^{\mathrm{b}}$

Seung-Hak Baek ${ }^{\mathrm{c}}$ (1)

${ }^{a}$ Department of Orthodontics, School of Dentistry, Kyungpook National University, Daegu, Korea

bPrivate Practice, Seoul, Korea

'Department of Orthodontics, School of Dentistry, Seoul National University, Seoul, Korea

\begin{abstract}
Objective: To investigate the three-dimensional (3D) surgical accuracy between virtually planned and actual surgical movements (SM) of the maxilla in twojaw orthognathic surgery. Methods: The sample consisted of 15 skeletal Class 111 patients who underwent two-jaw orthognathic surgery performed by a single surgeon using a virtual surgical simulation (VSS) software. The 3D cone-beam computed tomography (CBCT) images were obtained before (T0) and after surgery (T1). After merging the dental cast image onto the TO СBCT image, VSS was performed. SM were classified into midline correction (anterior and posterior), advancement, setback, anterior elongation, and impaction (total and posterior). The landmarks were the midpoint between the central incisors, the mesiobuccal cusp tip (MBCT) of both first molars, and the midpoint of the two MBCTs. The amount and direction of SM by VSS and actual surgery were measured using 3D coordinates of the landmarks. Discrepancies less than $1 \mathrm{~mm}$ between VSS and T1 landmarks indicated a precise outcome. The surgical achievement percentage (SAP, [amount of movement in actual surgery/ amount of movement in VSS] $\times 100)(\%)$ and precision percentage (PP, [number of patients with precise outcome/number of total patients] $\times 100)(\%)$ were compared among SM types using Fisher's exact and Kruskal-Wallis tests. Results: Overall mean discrepancy between VSS and actual surgery, SAP, and PP were 0.13 $\mathrm{mm}, 89.9 \%$, and $68.3 \%$, respectively. There was no significant difference in the SAP and PP values among the seven SM types (all $p>0.05$ ). Conclusions: VSS could be considered as an effective tool for increasing surgical accuracy.
\end{abstract}

[Korean J Orthod 2020;50(5):293-303]

Key words: Three-dimensional analysis, Virtual orthognathic surgery, Surgical movements of the maxilla, Two-jaw orthognathic surgery

Received April 10, 2020; Revised June 7, 2020; Accepted June 8, 2020.

Corresponding author: Seung-Hak Baek.

Professor, Department of Orthodontics and Dental Research Institute, School of Dentistry, Seoul National University, 101 Daehak-ro, Jongno-gu, Seoul 03080, Korea.

Tel +82-2-2072-3952 e-mail drwhite@unitel.co.kr

How to cite this article: Hong M, Kim MJ, Shin HJ, Cho HJ, Baek SH. Three-dimensional surgical accuracy between virtually planned and actual surgical movements of the maxilla in two-jaw orthognathic surgery. Korean J Orthod 2020;50:293-303.

(C) 2020 The Korean Association of Orthodontists.

This is an Open Access article distributed under the terms of the Creative Commons Attribution Non-Commercial License (http://creativecommons.org/licenses/by-nc/4.0) which permits unrestricted non-commercial use, distribution, and reproduction in any medium, provided the original work is properly cited. 


\section{INTRODUCTION}

Precise surgical positioning of the maxilla is critical for ensuring facial esthetics and functional occlusion in patients undergoing two-jaw orthognathic surgery. ${ }^{1-3}$ Cephalometric measurements and model surgery are performed to set up accurate and realistic surgery planning and to assess the actual surgical outcomes. ${ }^{1-6}$ However, the conventional model surgery procedure is known to involve a substantial laboratory burden and numerous errors during impression-taking, stone-setting, facebow transfer, model-mounting, and model surgery. ${ }^{7,8}$

With the recent development of 3-dimensional (3D) technology, fabrication of digital orthodontic study models, 3D analysis and superimposition of cone-beam computed tomography (CBCT) images, and virtual surgical simulation (VSS) and fabrication of surgical splints using 3D printing are available for every-day clinical situations. ${ }^{7,9-17}$ Lin and Lo, ${ }^{18}$ in an overview of the stateof-art methods for 3D computer-assisted technology in orthognathic surgery, enumerated current commercially available VSS software including Mimics (Materialise NV, Leuven, Belgium), SimPlant OMS (Materialise NV), Dolphin Imaging (Dolphin Imaging and Management Solutions, Chatsworth, CA, USA), and Maxilim (Medicim NV, Mechelen, Belgium). The advantages of 3D-VSS technology in orthognathic surgery include: (1) 3D measurements of the anatomical landmarks or structures, especially in the transverse dimension, which is difficult to perform with two-dimensional (2D) data; (2) repeatable simulation for alternative surgical plans using the same patients' data; (3) manufacturing the surgical splints according to virtual surgical plan; (4) precise evaluation of the actual surgical outcome using superimposition of the 3D volume images before and after orthognathic surgery. ${ }^{7,9-18}$ However, assessment of the accuracy of 3D computer-assisted orthognathic surgery using postoperative evaluation is still controversial.

Choi et al. ${ }^{4}$ investigated surgical accuracy in skeletal Class 111 patients who were treated with two-jaw surgery by a single surgeon and reported that the mean discrepancy between the surgical planning and actual surgical result was less than $1 \mathrm{~mm}$ in all surgical movement groups. However, they used 2D lateral cephalograms taken before and after surgery. Therefore, it is necessary to investigate the 3D surgical accuracy of maxillary repositioning according to diverse surgical movement types in two-jaw orthognathic surgery cases in the sagittal, vertical, and transverse planes. However, to the best of our knowledge, no previous study has assessed the precision of virtual surgical planning in diverse surgical movements. Thus, the purpose of this retrospective study was to investigate the 3D surgical accuracy between virtually planned and actual surgical movements of the maxilla in two-jaw orthognathic surgery.

\section{MATERIALS AND METHODS}

\section{Subjects}

The study population consisted of 15 Korean young adult patients with skeletal Class 111 malocclusion (9 males and 6 females; mean age, 22.3 years) between January 2019 and August 2019. To avoid sampling biases, we restricted study inclusion to patients: (1) who had the same skeletal pattern (Class 111 malocclusion) to avoid skeletal pattern-related bias, (2) who underwent the same surgical method (Le Fort 1 osteotomy and bilateral sagittal split ramus osteotomy [BSSRO]), (3) who were treated by a single surgeon (M.J.K.) to avoid surgeon-related bias, and (4) whose surgical planning procedures were completely virtually simulated using the ON3D software (3D ONS Inc., Seoul, Korea).

We excluded patients (1) who had skeletal Class 1 and 11 malocclusion, (2) who underwent one-jaw surgery, and (3) who had craniofacial deformity. This study was reviewed and approved by the Institutional Review Board of the Kyungpook National University Dental Hospital (KNUDH-2020-01-01-00).

\section{Obtainment of three-dimensional cone-beam computed tomography}

The 3D-CBCT scans were obtained $16.6 \pm 9.2$ days before surgery (TO) and two days after surgery (interposed with a final splint, T1) with PaX-Zenith3D (Vatech, Seoul, Korea). These images were used for virtual surgical planning and analyzing the actual surgical outcomes using the ON3D software.

\section{Reorientation of $\mathrm{CBCT}$ images (Figure 1)}

The 3D-volume images were automatically re-orientated using the software with the protocol reported by Cho. ${ }^{9}$ The unique feature of the ON3D software is presence of the "automatic digitization of the landmarks" function and the "automatic re-orientation" function that uses five landmarks, namely, the nasion $(\mathrm{N})$, right and left fronto-zygomatic points, right porion (Po), and right orbitale (Or), which makes treatment planning, surgical simulation and evaluation of surgical outcome easy and simple.

For the first cranial base reference plane, the nasofronto-zygomatic plane was automatically constructed using $\mathrm{N}$ point and the right and left fronto-zygomatic points. Then, the $\mathrm{N}$ point was registered as the origin $(0,0,0)$ of a Cartesian coordinate system. Hence, the $3 \mathrm{D}$-coordinate values $(\mathrm{x}, \mathrm{y}, \mathrm{z})$ of a specific landmark indicated its spatial location from the $\mathrm{N}(0,0,0)$. As the second reference plane, the right Frankfort horizontal (FH) plane was automatically established using the right 

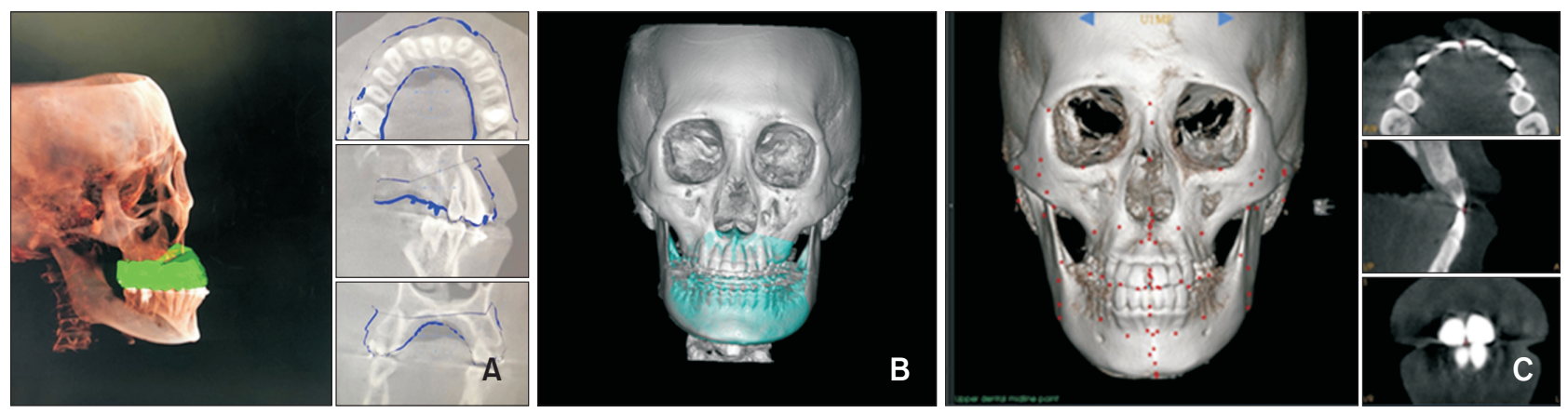

Figure 1. The process of merging between the scanned dentition and the three-dimensional cone-beam computed tomography (3D-CBCT) image and landmark digitization. A, Merging of the scanned dentition with 3D-CBCT image using three sectional views of the sagittal, frontal, and axial planes. B, Result of fine-tuned merging between dentition and 3D-CBCT images. C, Landmark digitization to identify the midpoint between the maxillary central incisors at the occlusal level (U1MP) and the mesiobuccal cusp tips (MBCTs) of the maxillary right and left first molar crowns using the three sectional views of the sagittal, frontal, and axial planes. The mean coordinate value from the two digitized MBCTs was automatically calculated by the ON3D software (3D ONS Inc., Seoul, Korea).

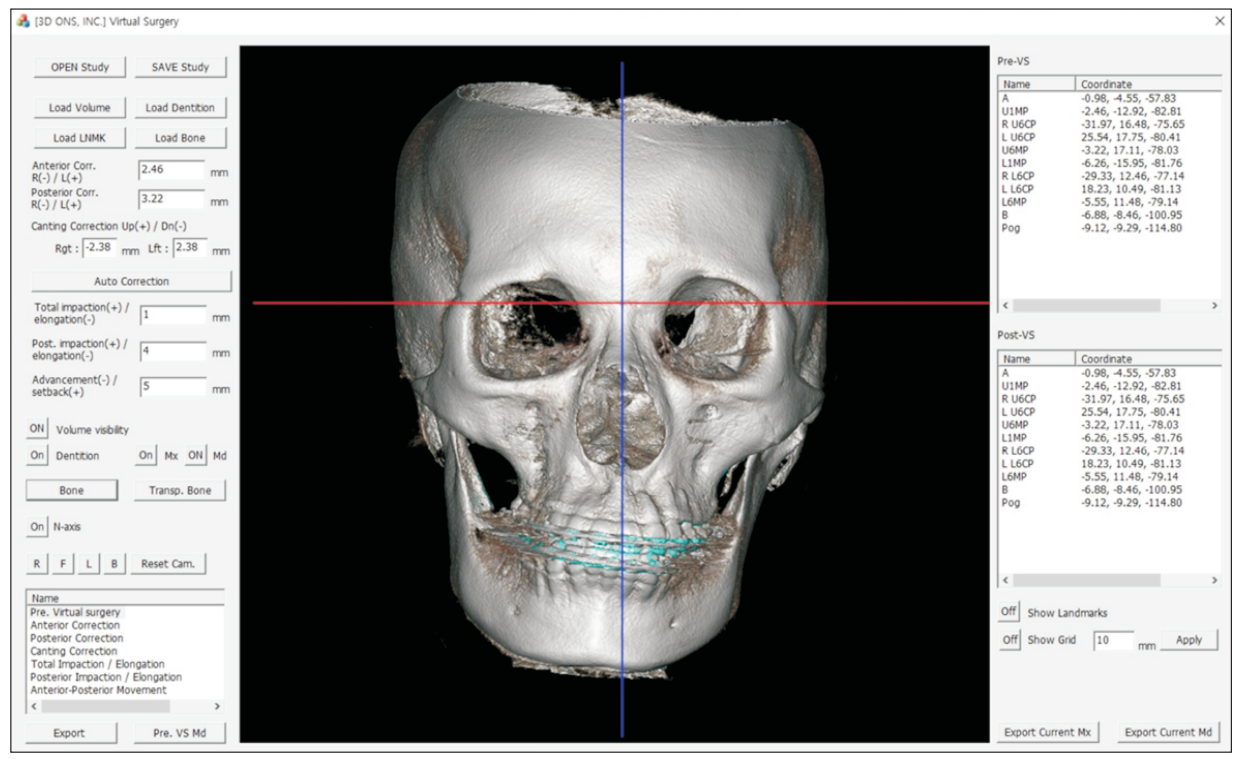

Figure 2. Virtual surgery modules of the ON3D software (3D ONS Inc., Seoul, Korea).

Po and Or. Basically, the amount of change in the 3Dcoordinate value $(x, y, z)$ of landmarks was used for surgical precision analysis between virtual planning and $\mathrm{T} 1$ $3 \mathrm{D}$ volume images.

\section{Merging the TO scanned dentition data onto the TO three-dimensional volume image (Figure 1)}

After scanning of the T0 dental cast using a scanner (TRIOS 3 Mono; 3Shape, Copenhagen, Denmark), the standard tessellation language (STL) files of the dentition were merged onto the T0 3D-volume image in the following two steps. First, three points (usually the central incisor tip and the mesiobuccal cusp tips [MBCTs] of both first molars in the maxilla) marked on both 3Dvolume images and the scanned dentition image were matched using the software. Second, further sophisticated merging of the dentition data with 3D-volume image was adjusted meticulously on the sectional views of the sagittal, frontal, and axial planes.

\section{Virtual surgical simulation}

VSS was performed using the Virtual Surgery Module of the ON3D software (Figure 2). The step-by-step procedure is as follows (Figure 3): step 1, correction of the anterior midline of the maxilla; step 2, correction of the posterior midline of the maxilla; step 3, correction of the vertical asymmetry (canting) of the maxilla; step 4, correction of the vertical discrepancy of the maxilla; step 5 , correction of the maxillary occlusal plane in the sagittal plane; step 6, correction of the sagittal discrepancy 


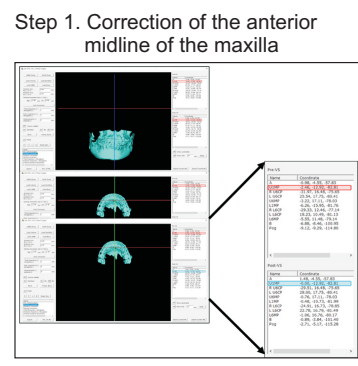

Step 2. Correction of the posterior midline of the maxilla

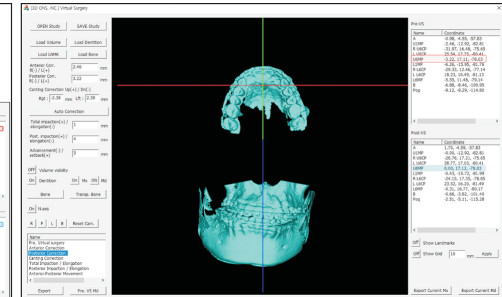

Step 3. Correction of the vertical asymmetry (canting) of the maxilla

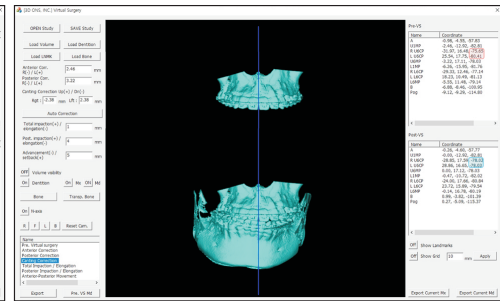

Step 6. Correction of the sagittal discrepancy of the maxilla
Step 4. Correction of the vertical discrepancy of the maxilla

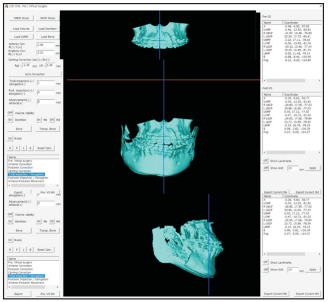

Step 5. Correction of the maxillary occlusal plane in the sagittal plane
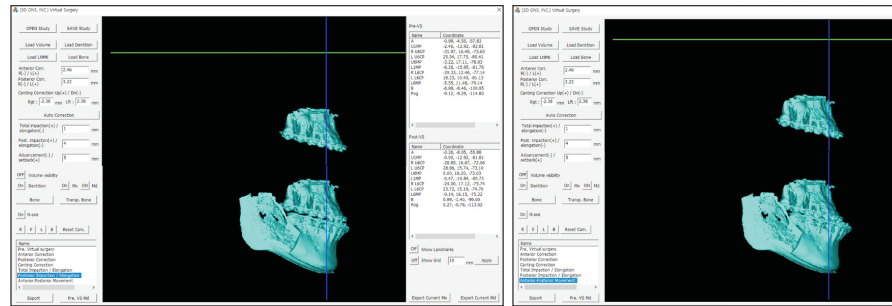

Step 7. Mandibular surgical correction simulation

Figure 3. The step-by-step procedure of virtual surgical simulation. Step 1, Correction of the anterior midline of the maxilla. Step 2, Correction of the posterior midline of the maxilla. Step 3, Correction of the vertical asymmetry (canting) of the maxilla. Step 4, Correction of the vertical discrepancy of the maxilla. Step 5, Correction of the maxillary occlusal plane in the sagittal plane. Step 6, Correction of the sagittal discrepancy of the maxilla. Step 7, Correction of the mandible.

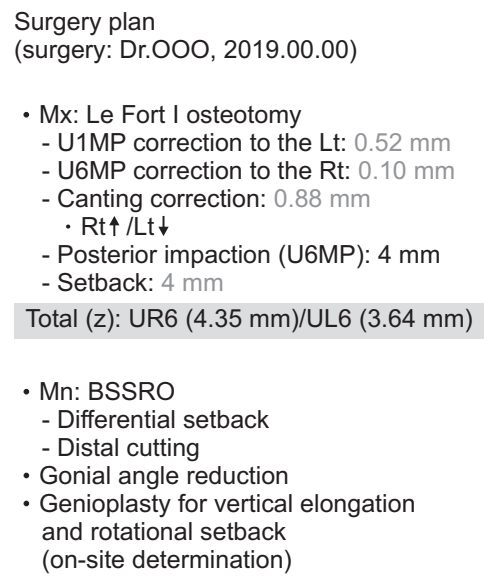

\begin{tabular}{|c|c|c|c|c|c|c|c|}
\hline & & & |3D & ual s & ation & & \\
\hline & & Pre- & & & & ost-VS & \\
\hline A & & -1.07 & -2.76 & -55.30 & -0.40 & -2.31 & -54.47 \\
\hline U1MP & & -0.52 & -9.31 & -75.36 & -0.00 & -5.31 & -75.36 \\
\hline U6CP & $\mathrm{R}$ & -25.59 & 14.46 & -73.39 & -25.69 & 17.00 & -69.04 \\
\hline & $L$ & 25.14 & 13.89 & -72.52 & 25.04 & 17.83 & -68.88 \\
\hline U6MP & & -0.22 & 14.17 & -72.95 & -0.32 & 17.41 & -68.96 \\
\hline L1MP & & 0.60 & -11.88 & -75.33 & 0.14 & -1.25 & -71.96 \\
\hline L6CP & $\mathrm{R}$ & -21.54 & 13.02 & -73.93 & -21.88 & 23.34 & -67.23 \\
\hline & $L$ & 22.11 & 13.85 & -73.31 & 21.78 & 24.06 & -67.48 \\
\hline L6MP & & 0.28 & 13.44 & -73.62 & -0.05 & 23.70 & -67.36 \\
\hline B & & 1.23 & -9.13 & -88.88 & 0.48 & 3.04 & -85.12 \\
\hline Pog & & 1.34 & -13.41 & -103.36 & 0.26 & 0.44 & -100.00 \\
\hline Post & $\mathrm{R}$ & -44.48 & 58.57 & -74.73 & -42.53 & 58.73 & -74.57 \\
\hline & $L$ & 45.91 & 60.59 & -74.11 & 44.09 & 60.87 & -74.00 \\
\hline
\end{tabular}

Figure 4. An example of surgical plan and coordinate values of the selected landmarks at the pre- and post-virtual surgery (VS).

Mx, maxilla; Mn, mandible; Lt, left; Rt, right; BSSRO, bilateral sagittal split ramus osteotomy; U1MP, the midpoint between the maxillary central incisors at the occlusal level; U6MP, the midpoint between the two mesiobuccal cusp tips of the maxillary right and left first molar crowns; UR6, the mesiobuccal cusp tip of the maxillary right first molar crown; UL6, the mesiobuccal cusp tip of the maxillary left first molar crown; U6CP, the mesiobuccal cusp tip of the maxillary first molar crown; L1MP, the midpoint between the mandibular central incisors at the occlusal level; L6CP, the mesiobuccal cusp tip of the mandibular first molar crown; L6MP, the midpoint between the two mesiobuccal cusp tips of the mandibular right and left first molar crowns; A, A point; B, B point; Pog, pogonion; Go, gonion. 
of the maxilla; and step 7, mandibular surgical correction simulation. Then, the result of VSS was adjusted indetail iteratively until the best virtual surgical outcome in the 3D-coordinates was obtained. The final 3Dcoordinate values of the landmarks were set as the goals of the actual surgical correction (Figure 4).

\section{Three-dimensional printing of surgical splints}

After VSS of the maxilla and mandible, intermediate and final splints were designed using computer aided design (CAD) software (Geomagic Freeform; 3D Systmes, Rock Hill, SC, USA). Then, these splints were fabricated with a 3D printing machine (Form 2; Formlabs, Somerville, MA, USA). According to Mangano et al's study, ${ }^{19}$ Form 2 showed the one of the best performances in both linear and diameter measurements. Therefore, they reported that the error of this $3 \mathrm{D}$ printer was compatible with its clinical use.

Formlabs clear resin (Formlabs) was used for fabrication of the intermediate and final wafers. In the concept and the technique used by surgeon (M.J.K), the final wafer was removed after fixation of the mandible, and two triangle vertical elastics (3/16 inch, 4 ounce) were placed between the maxillary canines, and mandibular canines and premolars. Two days after surgery, the final surgical wafer was used one more time for taking postoperative CBCT scans.

\section{Landmarks}

The landmarks used for measurement were the midpoint between the maxillary central incisors at the occlusal level (U1MP), the MBCTs of the maxillary right and left first molar crowns, and the midpoint between the two MBCTs (U6MP). In order to identify the MBCT of the maxillary first molars from $\mathrm{T} 0$ and $\mathrm{T} 1 \mathrm{CBCT}$ images, we used the function of three sectional views shown in Figure 1. Scrolling back and forth in three different dimensions was practically helpful for understanding the anatomical structure around the target areas or points. Additionally, intraoral photographs and study models were also utilized as references.

Since the 0N3D software automatically calculates the mean coordinate value from the two digitized MBCTs of the maxillary right and left first molar crowns, U6MP is not an actually digitized point and is presented on the screen of the user interface during surgical planning, VSS, and evaluation of surgical outcomes for clinicians. For example, in Figure 3, step 2 presents how the landmark value of U6MP is used for correction of the posterior midline of the maxilla.

\section{Superimposition of the T0 and T1 three-dimensional volume images \\ After automatic superimposition of the T0 and T1 3D-}

volume images using the anterior cranial base, ${ }^{20,21}$ the landmarks from the surgically unchanged area of the T0 3Dvolume image were imported to the T13D-volume image.

\section{Validation of landmark identification}

Second identification of the landmark was performed with a 1-month interval for five cases randomly selected by a single operator (H.J.S). Intra-class correlation coefficient (ICC) values for landmark identification reliability were calculated. Since the ICC values for all landmark identifications were above 0.86 ( $p<0.05$, appendix), the first set of measurements was used for further analysis.

\section{Types of surgical movements of the maxilla}

The surgical movements of the maxilla were classified as anterior midline correction, posterior midline correction, maxillary advancement, maxillary setback, maxillary total impaction, maxillary anterior elongation, and maxillary posterior impaction.

Anterior midline correction, maxillary advancement, maxillary setback, maxillary total impaction, and maxillary anterior elongation were evaluated using U1MP, while posterior midline correction and maxillary posterior impaction were assessed using U6MP.

\section{Measurement variables representing surgical accuracy}

For quantitative assessment of the surgical movement of the maxilla, the T0 and T1 3D-coordinate values of the landmarks on the superimposed volume images were calculated. The amount and direction of surgical movement of the landmarks by VSS and actual surgery in the 3D coordinates were measured. A discrepancy between VSS and T1 landmarks less than $1 \mathrm{~mm}$ was considered to indicate a precise outcome. ${ }^{4}$ The surgical achievement percentage (SAP, [amount of movement in actual surgery/amount of movement in virtual surgery] $\times 100)(\%)$ and precision percentage (PP, [number of patients who showed a precise outcome/number of total patients] $\times 100)(\%)$ were calculated in each surgical movement type of the maxilla. ${ }^{4}$ Therefore, the surgical accuracy was evaluated using three measurement variables, i.e., the discrepancies between VSS and T1, SAP, and PP.

\section{Statistical analysis}

Fisher's exact test and Kruskal-Wallis test were performed for statistical analysis using IBM SPSS Statistics, ver. 23.0 (IBM Corp., Armonk, NY, USA). $p$-values less than 0.05 were considered statistically significant.

\section{RESULTS}

Cephalometric characteristics of the samples (Table 1)

At the T0 stage, the patients exhibited a normally positioned maxilla (SNA, 82.3 ${ }^{\circ}$ and A-N perp, $-0.3 \mathrm{~mm}$ ), 
Table 1. Comparison of the cephalometric variables at pre- (T0) and post-surgery (T1)

\begin{tabular}{|c|c|c|c|c|c|}
\hline \multirow{2}{*}{ Cephalometric variable } & \multicolumn{2}{|c|}{ T0 stage } & \multicolumn{2}{|c|}{ T1 stage } & \multirow{2}{*}{$p$-value } \\
\hline & Mean & SD & Mean & SD & \\
\hline SNA $\left({ }^{\circ}\right)$ & 82.3 & 4.4 & 83.2 & 3.5 & 0.246 \\
\hline $\operatorname{SNB}\left(^{\circ}\right)$ & 84.3 & 4.2 & 79.8 & 3.8 & $0.000^{* * *}$ \\
\hline $\operatorname{ANB}\left({ }^{\circ}\right)$ & -2.0 & 2.5 & 3.4 & 1.0 & $0.000^{* * *}$ \\
\hline A-N perp (mm) & -0.3 & 4.7 & 1.3 & 2.9 & 0.073 \\
\hline Pog-N perp (mm) & 5.4 & 8.7 & -0.9 & 6.0 & $0.001^{* * *}$ \\
\hline $\operatorname{FMA}\left(^{\circ}\right)$ & 25.8 & 7.8 & 29.6 & 5.3 & $0.005^{* *}$ \\
\hline $\mathrm{U} 1$ to $\mathrm{FH}\left({ }^{\circ}\right)$ & 112.9 & 6.6 & 106.4 & 4.2 & $0.001^{* *}$ \\
\hline $\operatorname{IMPA}\left({ }^{\circ}\right)$ & 85.4 & 6.7 & 83.1 & 7.3 & 0.110 \\
\hline
\end{tabular}

The samples consisted of 15 Korean young adult patients with skeletal Class III malocclusion (9 males and 6 females; mean age, 22.3 years).

Paired $t$-test was performed.

SNA, Sella-nasion-point A angle; SNB, sella-nasion-point B angle; ANB, point A-nasion-poing B angle; A-N perp, point A-nasion perpendicular line; Pog-N perp, pogonion-nasion perpendicular line; FMA, Frankfort horizontal plane to mandibular plane angle; U1 to FH, maxillary incisor inclination to Frankfort horizontal plane; IMPA, incisor mandibular plane angle; SD, standard deviation.

${ }^{* *} p<0.01,{ }^{* * *} p<0.001$.

protrusive mandible (SNB, $84.3^{\circ}$ and Pog-N perp, 5.4 $\mathrm{mm}$ ), normodivergent pattern (FMA, 25.8 $)$, and linguoversed mandibular incisor (IMPA, $85.4^{\circ}$ ).

At the T1 stage, the patients showed significant mandibular setback (SNB, $84.3^{\circ}$ to $79.8^{\circ}, p<0.001$; Pog-N perp, $5.4 \mathrm{~mm}$ to $-0.9 \mathrm{~mm}, p<0.001$ ), increase in FMA $\left(25.8^{\circ}\right.$ to $\left.29.6^{\circ}, p<0.01\right)$, linguoversion of the maxillary incisor ( $112.9^{\circ}$ to $106.4^{\circ}, p<0.01$ ), and correction of a Class 111 relationship (ANB, $-2.0^{\circ}$ to $3.4^{\circ}, p<0.001$ ).

\section{Surgical movement types of the maxilla (Table 2)}

Among the seven types of surgical movements, anterior midline correction was the most frequently performed one $(93.3 \%, n=14 / 15)$, followed by posterior midline correction $(86.7 \%, n=13 / 15)$, maxillary posterior impaction $(80.0 \%, n=12 / 15)$, maxillary setback $(66.7 \%$, $n=10 / 15)$, maxillary anterior elongation $(40.0 \%, n=$ $6 / 15)$, maxillary total impaction (33.3\%, $n=5 / 15)$, and maxillary advancement $(20.0 \%, n=3 / 15)$.

Comparison of surgical accuracy among the surgical movement types of the maxilla surgical accuracy in the overall dimensions (Tables 2 and 3 )

Although the overall mean discrepancy between the virtually planned and actual movement was $0.1 \mathrm{~mm}$, it differed significantly according to the surgical movement types of the maxilla $(p<0.05)$; In particular, the mean discrepancy values of maxillary anterior elongation showed a significant difference from those of maxillary posterior impaction (-0.85 mm [overcorrection]) vs. 0.65 $\mathrm{mm}$ [undercorrection], $p<0.05)$. However, overall SAP was $89.9 \%$ and overall PP was $68.3 \%$ without significant difference among surgical movement types (both $p$ $>0.05)$.

\section{Surgical accuracy in the transverse dimension (Tables 2 and 3)}

In terms of anterior midline correction, the discrepancy between virtually planned and actual movement was $-0.1 \mathrm{~mm}$, indicating an almost perfect match. Although SAP was $103.4 \%$, PP was $64.3 \%$. In terms of posterior midline correction, the discrepancy between virtually planned and actual movement was $0.0 \mathrm{~mm}$, indicating an almost perfect match. Although SAP was 95.8\%, PP was $69.2 \%$.

\section{Surgical accuracy in the sagittal dimension (Tables 2 and 3)}

In terms of maxillary advancement, discrepancy between virtually planned and actual movement was 1.2 $\mathrm{mm}$, indicating clinically meaningful undercorrection. SAP was $65.5 \%$ and PP was only 33.3\%. In terms of maxillary setback, discrepancy between virtually planned and actual movement was $0.1 \mathrm{~mm}$, indicating an almost perfect match. Both SAP and PP were as high as $89.8 \%$ and $80.0 \%$, respectively.

\section{Surgical accuracy in the vertical dimension (Tables 2 and 3)}

In terms of maxillary total impaction, discrepancy between virtually planned and actual movement was $0.4 \mathrm{~mm}$, indicating slight undercorrection. Although 


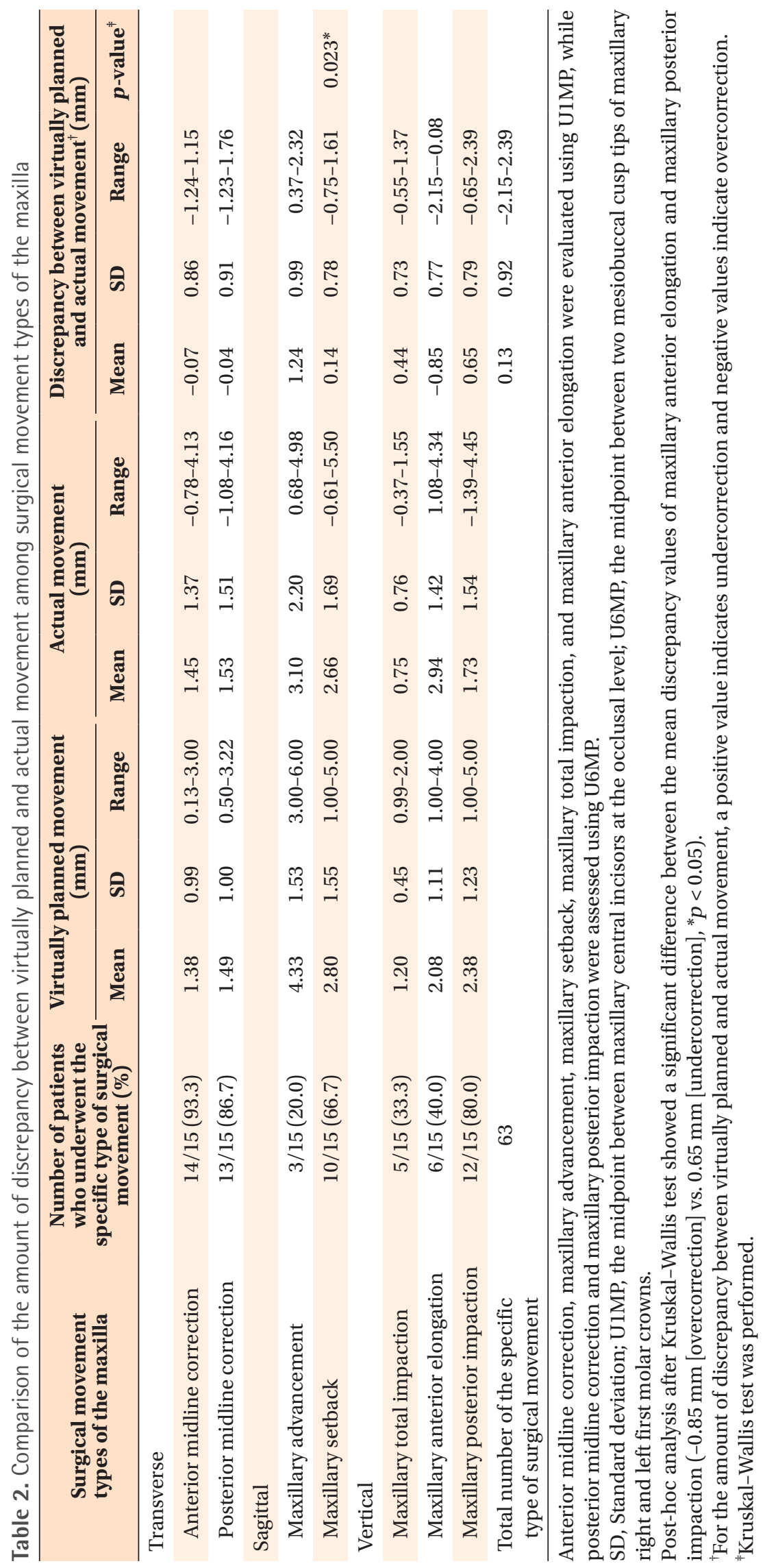




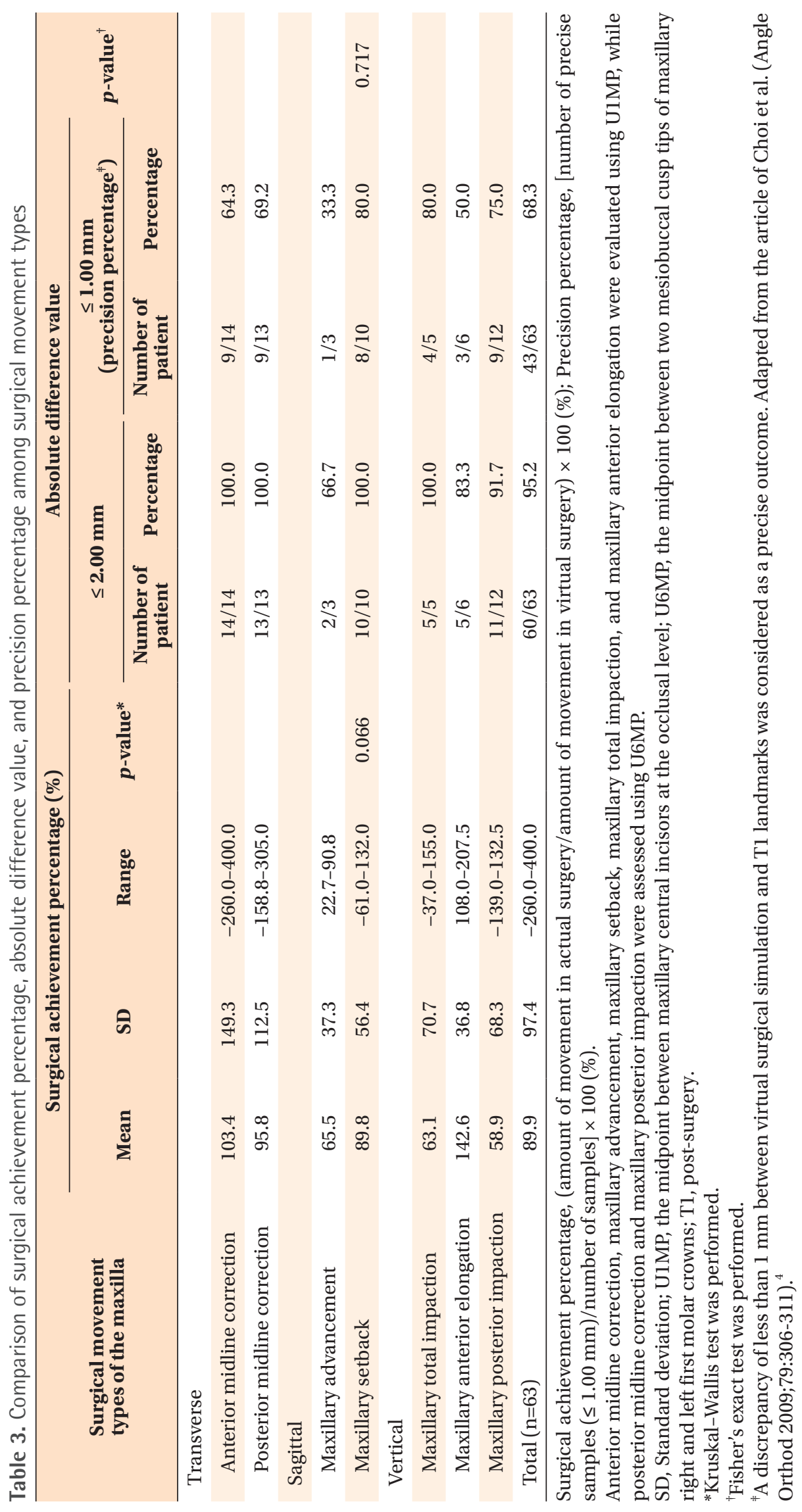


maxillary total impaction exhibited a high degree of PP $(80.0 \%)$, it showed a moderate degree of SAP $(63.1 \%)$. In terms of maxillary anterior elongation, discrepancy between virtually planned and actual movement was $-0.9 \mathrm{~mm}$, indicating slight overcorrection. Since SAP was $142.6 \%$, PP was only $50.0 \%$. In terms of maxillary posterior impaction, the discrepancy between the virtually planned and actual movement was $0.7 \mathrm{~mm}$, indicating slight undercorrection. Although maxillary posterior impaction exhibited a moderate-to-high degree of PP $(75.0 \%)$, it presented a moderate degree of SAP $(58.9 \%)$.

\section{DISCUSSION}

The 3D virtual surgical planning of orthognathic surgery allows orthodontists and surgeons to review the possible surgical options, which might produce more accurate and stable surgical outcome compared to conventional surgical planning. However, whether the surgical outcome obtained by a new methodology is compatible to the conventional one should be confirmed.

\section{Surgical accuracy in the overall dimensions (Tables 2 and 3)}

Overall mean discrepancy between virtually planned and actual surgical movement, SAP, and PP were 0.13 $\mathrm{mm}, 89.9 \%$, and $68.3 \%$, respectively. There was no significant difference in the distribution of SAP and PP among surgical movement types (all $p>0.05$ ). In the present study, $95.2 \%$ of the sample showed less than 2 $\mathrm{mm}$ of discrepancy and $68.3 \%$ showed less than $1 \mathrm{~mm}$ of discrepancy between the virtually planned and actual positions (Table 3). These results showed higher accuracy than those of the previous studies. ${ }^{1,2}$ Although Choi et al. ${ }^{4}$ and Gil et al. ${ }^{3}$ reported that $100 \%$ of their sample showed less than $1 \mathrm{~mm}$ of discrepancy, they used measurements of 2D cephalograms and model surgery.

Although this study showed a higher PP than Choi et al. ${ }^{4}$ in maxillary setback (80.0\% vs. $69.2 \%$ ) and maxillary posterior impaction $(75.0 \%$ vs. $69.0 \%)$, the other surgical movement types showed much lower PPs as follows: maxillary advancement, $33.3 \%$ vs. $87.5 \%$; maxillary anterior elongation, $50.0 \%$ vs. $83.3 \%$ (Table 2). These differences might be attributable to differences in the study design and methods as follows: First, the reference planes used in this study (naso-fronto-zygomatic plane and right $\mathrm{FH}$ plane) were different from those used by Choi et al. ${ }^{4}$ (the horizontal reference line [which was angulated $7^{\circ}$ clockwise from 'Sella' to 'Nasion' line] and the vertical reference line [which was perpendicular to the horizontal reference line]). Second, the distance between the virtually planned and actual landmarks in 3D CBCT images would be different from that in 2D lateral cephalograms.

\section{Surgical accuracy in the transverse dimension (Tables 2 and 3)}

The present study exhibited that anterior midline correction and posterior midline correction had an almost perfect match between the virtually planned and actual movements $(-0.07 \mathrm{~mm}$ and $-0.04 \mathrm{~mm})$ and showed very high degree of SAP (103.4\% and 95.8\%) and moderate degree of PP (64.3\% and 69.2\%). However, previous studies reported relatively higher discrepancies of as much as $0.15-0.8 \mathrm{~mm}$ in the transverse dimension compared to this study. ${ }^{12,14,22,23}$

\section{Surgical accuracy in the sagittal dimension (Tables 2 and 3)}

Maxillary setback showed a tendency to yield lower mean values of discrepancy between virtually planned and actual movement, higher SAP, and higher PP compared to maxillary advancement $(0.14 \mathrm{~mm}, 89.8 \%$, and $80.0 \%$ vs. $1.24 \mathrm{~mm}, 65.5 \%$, and 33.3\%). However, Choi et al., ${ }^{4}$ in their 2D cephalometric analysis, reported almost perfect match between virtually planned and actual movement, high SAP, and moderate PP in both maxillary advancement $(-0.05 \mathrm{~mm}, 100.8 \%, 87.5 \%)$ and maxillary setback $(-0.02 \mathrm{~mm}, 107.1 \%, 69.2 \%)$. Previous 3D studies reported a variety of discrepancies in the anteroposterior direction from $0.25 \mathrm{~mm}$ to $8 \mathrm{~mm}$ at the maxillary incisor. ${ }^{12,14,17,22-24}$ Since this study had only three cases of maxillary advancement, further studies with larger sample sizes are necessary to investigate the reasons for these differences.

\section{Surgical accuracy in the vertical dimension (Tables 2 and 3)}

There was $0.7 \mathrm{~mm}$ of undercorrection in maxillary posterior impaction and $0.9 \mathrm{~mm}$ of overcorrection in maxillary anterior elongation. Maxillary posterior impaction showed a tendency for lower SAP and higher PP compared to maxillary anterior elongation without statistical difference $(58.9 \%$ vs. $142.6 \%, 75.0 \%$ vs. $50.0 \%$, all $p>0.05$ ). The reason might be due to difference in the bony contact and support area between the anterior and posterior maxilla, and the difficulty in management of the premature bony contact in the posterior maxilla. Previous 3D studies reported a mean discrepancy value of 0.5 to $1.85 \mathrm{~mm}$ for the vertical surgical movement of the maxilla. ${ }^{12,14,20-22}$ In addition, maxillary total impaction showed lower discrepancy between virtually planned and actual movement (0.44 $\mathrm{mm}$ undercorrection), moderate SAP (63.1\%), and high PP (80.0\%).

Although this study might provide meaningful clinical data for the 3D surgical accuracy of the maxillary repositioning with respect to the 3D coordinates between the VSS and actual surgery, its limitations included the small sample size due to the use of newly developed software 
(ON3D). Since the surgically planned position of the maxilla dictates the postoperative mandibular position, further studies with large sample sizes and multi-center study designs are necessary to investigate the surgical accuracy of mandibular repositioning in diverse types of malocclusion.

\section{CONCLUSION}

Virtual surgery planning could be considered as an effective tool for increasing surgical accuracy in terms of mean discrepancy between virtually planned and actual movements, SAP and PP.

\section{CONFLICTS OF INTEREST}

Dr. Heon Jae Cho is the CEO of 3D ONS Inc. and Dr. Myung-Jin Kim is a board member of 3D ONS Inc. However, this study did not receive any commercial, financial and material support.

\section{ACKNOWLEDGEMENTS}

This research was supported by Kyungpook National University Research Fund, 2017.

\section{REFERENCES}

1. Jacobson R, Sarver DM. The predictability of maxillary repositioning in LeFort 1 orthognathic surgery. Am J Orthod Dentofacial Orthop 2002;122:142-54.

2. Semaan S, Goonewardene MS. Accuracy of a LeFort 1 maxillary osteotomy. Angle Orthod 2005;75:964-73.

3. Gil JN, Claus JD, Manfro R, Lima SM Jr. Predictability of maxillary repositioning during bimaxillary surgery: accuracy of a new technique. Int J Oral Maxillofac Surg 2007;36:296-300.

4. Choi JY, Choi JP, Baek SH. Surgical accuracy of maxillary repositioning according to type of surgical movement in two-jaw surgery. Angle Orthod 2009;79:306-11.

5. Stokbro K, Aagaard E, Torkov P, Bell RB, Thygesen T. Surgical accuracy of three-dimensional virtual planning: a pilot study of bimaxillary orthognathic procedures including maxillary segmentation. Int J Oral Maxillofac Surg 2016;45:8-18.

6. Gaber RM, Shaheen E, Falter B, Araya S, Politis C, Swennen GRJ, et al. A systematic review to uncover a universal protocol for accuracy assessment of 3-dimensional virtually planned orthognathic surgery. J Oral Maxillofac Surg 2017;75:2430-40.

7. Song KG, Baek SH. Comparison of the accuracy of the three-dimensional virtual method and the conventional manual method for model surgery and intermediate wafer fabrication. Oral Surg Oral Med Oral Pathol Oral Radiol Endod 2009;107:13-21.

8. De Riu G, Virdis PI, Meloni SM, Lumbau A, Vaira LA. Accuracy of computer-assisted orthognathic surgery. J Craniomaxillofac Surg 2018;46:293-8.

9. Cho HJ. A three-dimensional cephalometric analysis. J Clin Orthod 2009;43:235-52, discussion 235; quiz 273.

10. Metzger MC, Hohlweg-Majert B, Schwarz U, Teschner M, Hammer B, Schmelzeisen R. Manufacturing splints for orthognathic surgery using a threedimensional printer. Oral Surg Oral Med Oral Pathol Oral Radiol Endod 2008;105:e1-7.

11. Aboul-Hosn Centenero S, Hernández-Alfaro F. 3D planning in orthognathic surgery: CAD/CAM surgical splints and prediction of the soft and hard tissues results - our experience in 16 cases. J Craniomaxillofac Surg 2012;40:162-8.

12. Hernández-Alfaro F, Guijarro-Martínez R. New protocol for three-dimensional surgical planning and CAD/CAM splint generation in orthognathic surgery: an in vitro and in vivo study. Int $\mathrm{J}$ Oral Maxillofac Surg 2013;42:1547-56.

13. Stokbro K, Aagaard E, Torkov P, Bell RB, Thygesen T. Virtual planning in orthognathic surgery. Int J Oral Maxillofac Surg 2014;43:957-65.

14. Baan F, Liebregts J, Xi T, Schreurs R, de Koning M, Bergé $S$, et al. A new 3D tool for assessing the accuracy of bimaxillary surgery: the OrthoGnathicAnalyser. PLoS One 2016;11:e0149625.

15. Shaheen E, Sun Y, Jacobs R, Politis C. Three-dimensional printed final occlusal splint for orthognathic surgery: design and validation. Int $\mathrm{J}$ Oral Maxillofac Surg 2017;46:67-71.

16. Van den Bempt M, Liebregts J, Maal T, Bergé S, Xi $T$. Toward a higher accuracy in orthognathic surgery by using intraoperative computer navigation, 3D surgical guides, and/or customized osteosynthesis plates: a systematic review. J Craniomaxillofac Surg 2018;46:2108-19.

17. Tankersley AC, Nimmich MC, Battan A, Griggs JA, Caloss R. Comparison of the planned versus actual jaw movement using splint-based virtual surgical planning: how close are we at achieving the planned outcomes? J Oral Maxillofac Surg 2019;77:1675-80.

18. Lin HH, Lo LJ. Three-dimensional computer-assisted surgical simulation and intraoperative navigation in orthognathic surgery: a literature review. J Formos Med Assoc 2015;114:300-7.

19. Mangano FG, Admakin O, Bonacina M, Biaggini F, Farronato D, Lerner H. Accuracy of 6 desktop 3D printers in dentistry: a comparative in vitro study. Eur J Prosthodont Restor Dent 2020;28:75-85.

20. Choi JH, Mah J. A new method for superimposition 
of CBCT volumes. J Clin Orthod 2010;44:303-12.

21. Gkantidis N, Schauseil M, Pazera P, Zorkun B, Katsaros C, Ludwig B. Evaluation of 3-dimensional superimposition techniques on various skeletal structures of the head using surface models. PloS One 2015;10:e0118810.

22. Tucker S, Cevidanes LH, Styner M, Kim H, Reyes M, Proffit W, et al. Comparison of actual surgical outcomes and 3-dimensional surgical simulations. J Oral Maxillofac Surg 2010;68:2412-21.
23. Hsu SS, Gateno J, Bell RB, Hirsch DL, Markiewicz $\mathrm{MR}$, Teichgraeber JF, et al. Accuracy of a computeraided surgical simulation protocol for orthognathic surgery: a prospective multicenter study. J Oral Maxillofac Surg 2013;71:128-42.

24. Shehab MF, Barakat AA, AbdElghany K, Mostafa Y, Baur DA. A novel design of a computer-generated splint for vertical repositioning of the maxilla after Le Fort 1 osteotomy. Oral Surg Oral Med Oral Pathol Oral Radiol 2013;115:e16-25. 